\title{
Comparative evaluation of the doses received by the parotid glands as predictors of xerostomia be 3D-CRT, IMRT and VMAT irradiation techniques in local advanced nasopharynx cancer
}

\author{
Camil Mireștean ${ }^{1}$, Călin Gheorghe Buzea*1, Irina Butuc ${ }^{1}$, Alexandru Zara ${ }^{1}$, Dragoș \\ Teodor lancu ${ }^{2}$
}

${ }^{1}$ Regional Institute of Oncology, lasi, Romania, ${ }^{2}$ "Grigore T. Popa” University of Medicine and Pharmacy, lasi, Romania

\begin{abstract}
Xerostomia is commonly associated with the radio-chemotherapy treatment of the head and neck cancers. The risk increases with increasing doses received by the parotid. Severe xerostomia (defined as long-term salivary function of $<25 \%$ of baseline) may be avoided if at least one parotid gland receives less than 20 Gy. The combined treatment with cisplatin regarded as bringing a significant benefit in survival with concurrent radiotherapy is associated with increased risk of late toxicity. Intensity-modulated radiotherapy (IMRT) is considered the radio-therapeutic standard in the management of head and neck cancer. Purpose: to evaluate the possibility of modern techniques to reduce radiation doses to parotid glands compared to conventional 3DCRT radiotherapy even if the parotid glands are not delineated as organs at risk (OAR) and dosimetric constraints are not applied. Methods: For 10 locally advanced nasopharyngeal cancer cases treated by radiotherapy with curative intent using $3 \mathrm{D}-\mathrm{CRT}$ technique, alternative IMRT and VMAT plans were proposed without applying dosimetric constraints for parotid glands. Results: IMRT and VMAT techniques reduce the maximum dose (Dmax) and the mean dose (Dmean) for both parotid glands compared to the 3D-CRT technique. The treatment plans were comparatively analyzed in terms of doses received by both parotid glands. Conclusions: Modern radiotherapy techniques implementation can reduce the dose received by the parotids even in the absence of contouring them as organs at risk, reducing xerostomia and ensuring a better quality of life for the nasopharynx cancer radio-treated patients.
\end{abstract}

Keywords: nasopharyngeal cancer, intensity modulated radiation therapy, volumetric modulated arc therapy, xerostomia

\section{Introduction}

Radio-induced xerostomia is a side effect and often permanent of radiotherapy for head and neck cancer and a major cause of deterioration of patient quality of life [1].

Received: June 2017; Accepted after review: September 2017; Published: September 2017.

*Corresponding author: Călin Gheorghe Buzea, Regional Institute of Oncology, Henry Mathias Berthelot 2-4, Iasi, Romania. Tel: +40-753-675353, Fax: +40-232231132

E-mail: calinb2003@yahoo.com
The toxicity of radiotherapy on salivary gland itself is associated with decreased quantity and quality of saliva flow associated with symptomatic dry mouth syndrome. The dose received by the parotid glands (PG) has been proven as having predictive value of xerostomia, doses between 26-39 Gy being correlated to a significant decrease in salivary flow and doses between 60-70 Gy were associated with morphological changes associated with toxic effects irreversibility. Patient discomfort is often described as the tongue adherence to the soft palate during the night, being necessary intake of liquids. 
Patients often describe pain caused by cracks in the oral mucosa or retrograde infections of the salivary glands due to decreased salivary flow. Changing the $\mathrm{pH}$, chemical composition, physical properties (increase in viscosity) and enzymatic properties of the saliva has as consequence a favoring of fungal and bacterial infections and appearance and rapid progression of tooth decay through demineralization [2].

Implementation of the standard technique of IMRT in the treatment of nasopharyngeal cancer has led to better conformity of the target volume and a reduced dose received by radiosensitive organs and especially by the parotid glands $[3,4]$.

It is considered that the benefit of the quality of life of patients irradiated by IMRT technique through the attenuation of xerostomia is more common in more than 6 months after completion of radiotherapy [5].

\section{Methods}

All of the patients received the 3D-CRT treatment before IMRT and VMAT techniques were implemented and alternative IMRT and VMAT plans were created. All the treatment plans were done in the same computed tomography (CT) and structure set. The median age was 45, with 6 males and 4 females. The clinical stage distribution was T3 for six patients and T4 for four patients with N2-N3 node stage.

\section{Treatment protocol}

Head-and-neck tumors and their treatments can cause complex anatomical and functional deficits. A thorough initial assessment of tumor and patient factors including function, comorbidity and personal preference is essential to choose the optimal treatment pathway. Conformal radiotherapy of head-and-neck cancers requires knowledge of anatomy and patterns of spread of disease, which are often specific to each tumor site.

Patients underwent CT simulation using 3 $\mathrm{mm}$ thick slices, from $2 \mathrm{~cm}$ above the superior orbital ridge (to include the skull base) to the arch of the aorta inferiorly. Although intravenous contrast may help to define the cervical nodes, no oral or intravenous contrast was used at simulation. Reference marks are placed on the shell, at the CT visit, to aid verification. Patients were treated supine with head and shoulders immobilized in a Perspex shell or thermoplastic mask with at least five fixation points.

A single radiation oncologist completed contouring prior to commencement of the project. The GTV is first contoured on the planning CT using diagnostic images and clinical information. Particular attention is given to the para-pharyngeal space, and to the lateral pharyngeal lymph nodes. Retropharyngeal nodes $>5 \mathrm{~mm}$ and cervical nodes $>10 \mathrm{~mm}$ in short axis diameter are contoured as GTV. The GTV is expanded isotropically by $5 \mathrm{~mm}$ to form the CTV70 which is then edited to reflect natural tumor barriers.

Three CTVs are defined: a high dose CTV70 reflecting the clinically apparent disease; a high risk CTV66 reflecting the high risk of local spread in and adjacent to the nasopharynx; and a prophylactic CTV50 to treat at risk but clinically uninvolved nodes. The CTV70 is copied to form the CTV66 which is expanded and includes the whole nasopharynx, adjacent retropharyngeal lymph nodal regions, parapharyngeal space, pterygoid plates, pterygomaxillary fissures, floor of the sphenoid sinus, foramen lacerum and the posterior part of the nasal cavity (5 $\mathrm{mm}$ anterior to the GTV).

A CTV-PTV margin is applied (usually 3-5 $\mathrm{mm}$ ) based on measured set-up errors assuming no tumor motion.

The brain, brainstem and spinal cord were defined. The parotid glands, cochlea and optic apparatus were also contoured as critical structures since IMRT and VMAT is used and dose sparing may be possible. Also, we mention that parotid glands and cochlea were considered second degree priority OAR's, and the target volume was not compromised to spare them. Moreover, for the 10 patients, even the parotid glands were contoured, one did not impose dosimetric constraints on them as critical structures. For each parotid, $D_{\min }$, $D_{\max }$ and $D_{\text {mean }}$ were evaluated comparatively 
between IMRT, VMAT and 3D-CRT techniques.

All 3D-CRT, IMRT and VMAT plans were created prospectively using the unique set of contours for each patient. A standalone Eclipse (Varian, Palo Alto, CA) treatment planning system, installed on a Dell Precision T5500 computer, was used for all plan creation.
The treatment plans were based on a three phase treatment on nasopharynx (cavum), using the original planning CT and contoured volumes for each of the 10 patients (Figure 1). The CTV's consisted of the gross tumor volumes (GTV's) and the critical structures as defined above.
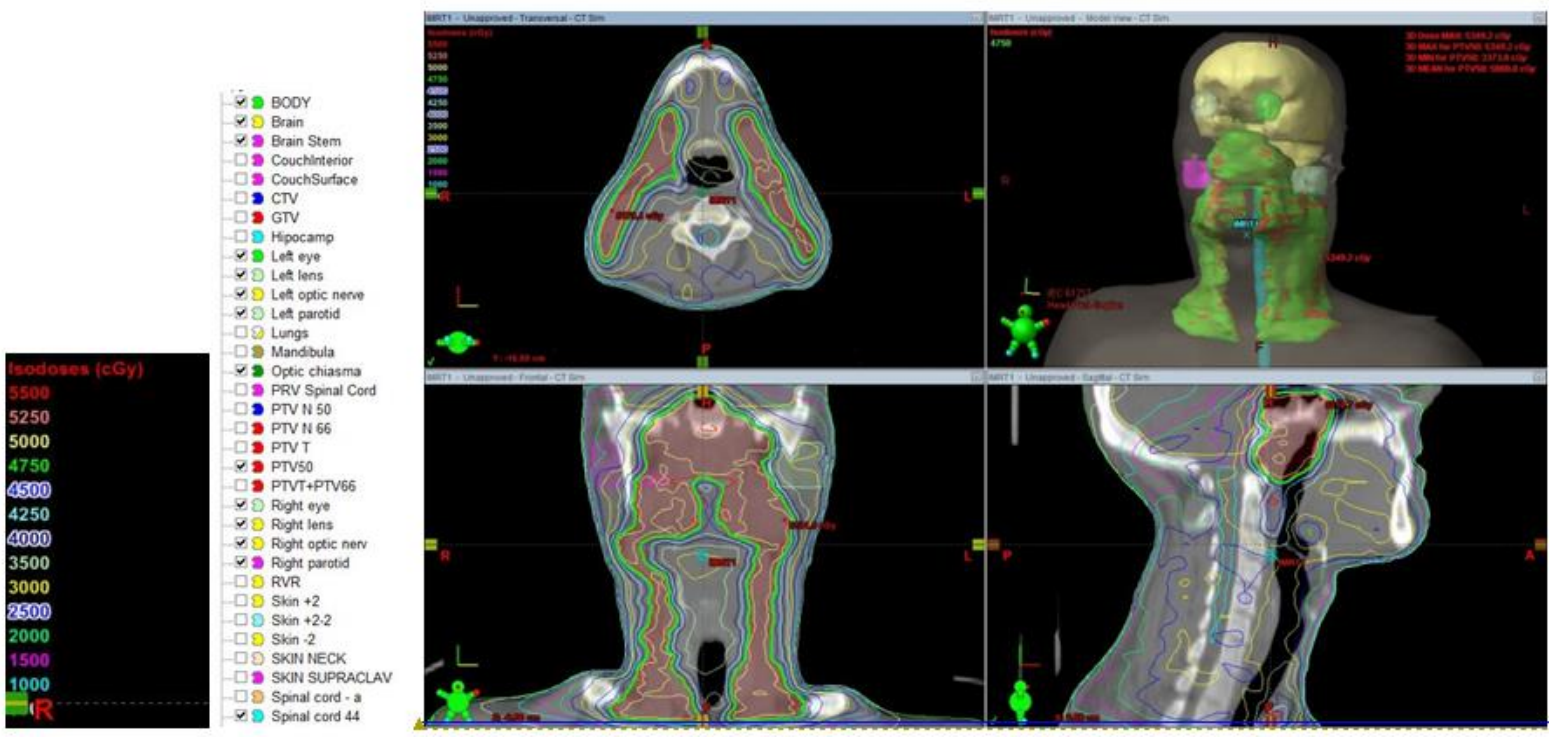

Fig. 1. Eclipse treatment plan window (top left-transversal view top right-modeview, bottom left - frontal view, bottom right - sagittal view) for one of the 10 patients. We also depicted the legend for the isodoses and the color codes for the contoured organs at risk

Each patient had one 3D-CRT, one IMRT and one VMAT plan created by the same medical physicist who has an extensive experience in 3D-CRT, IMRT and VMAT planning. The same dose constraints were used for creation of 3D-CRT, IMRT and VMAT plans (Table 1).

The total prescription dose on the PTV was based on a median dose of 70 Gy delivered in 35 fractions at 2 Gy/fraction treating daily, five days per week over seven weeks. It was divided sequentially as follow: TV50 (Phase I): a dose of 50 Gy delivered in 25 fractions at $2 \mathrm{~Gy} /$ fraction, PTV66 (Phase II): a dose of 16 Gy delivered in 8 fractions at 2 Gy/fraction and PTV70 (Phase III): a dose of 4 Gy delivered in 2 fractions at 2 Gy/fraction treating daily. Plans were normalized to ensure that the $95 \%$ isodose adequately covered the PTV and that the dose distribution was such that the minimum dose to $99 \%$ of the
PTV volume (D99\%) was greater than or equal to $95 \%$ of the prescribed dose $(50,66$ and 70 Gy, respectively) and the mean dose to the CTV was within 0,5 Gy of the prescribed dose. All plans were evaluated to ensure they met our institutional dose constraints outlined in Table 1. All 3D-CRT plans were composed of an adequate number of beam field arrangements using $6 \mathrm{MV}$ photons. MLC beam shaping, beam modifiers (eg. wedges) and field-in-field were employed as required to produce the most conformal dosimetry. All IMRT and VMAT plans were created using a 6MV photon beam applicable to a Varian Clinac iX (Varian Medical Systems, Palo Alto, CA) linear accelerator with a 120 leaf Millennium dynamic multi-leaf collimator (MLC). $6 \mathrm{MV}$ photons were used for IMRT and VMAT as this revealed by most published clinical studies as well as from current practice in our institution. 
IMRT plans were generated on the Eclipse Version 11.0.31 treatment planning software (Varian Medical Systems, Palo Alto, CA) using a 5-9 beam multi-field technique. The number of beams, and their position were automatically calculated using the built in Beam Angle Optimization 11.0.31 software facility, or were set up manually, according to the complexity of the target volume. The initial optimization parameters and their priorities were established according to our institutional optimization protocol and then adjusted as required to achieve the dose constraints (Table 1) using a minimum of 100 iterations. A Normal Tissue Objective was encompassed and the default smoothing parameters were applied to help reduce hotspots outside the target volume and the total number of monitor units.

Table 1. Clinical dosimetric constraints used in planning 3D-CRT/IMRT/VMAT

\begin{tabular}{|c|c|c|c|}
\hline & Dose to $0.1 \mathrm{~cm}^{3}$ & Dose to $<1 \mathrm{~cm}^{3}$ & Mean dose \\
\hline Spinal cord & 46 Gy & 45 Gy & \\
\hline Brainstem & 55 Gy & $54 \mathrm{~Gy}$ & $40 \mathrm{~Gy}$ \\
\hline Brain & & $60 \mathrm{~Gy}$ & \\
\hline Whole parotid & & & 24 Gy-if aiming to preserve function \\
\hline Mandible & & 74 Gy & $<33 \%$ to receive $>65$ Gy \\
\hline Larynx & & & $\begin{array}{c}\text { Aim for } 50 \text { Gy but do not allow to compromise ptv } \\
\text { coverage }\end{array}$ \\
\hline Lens & & & $<6-8 \mathrm{~Gy}$ \\
\hline Retina & 50 Gy & & 45 Gy. If greater consent for possible visual loss \\
\hline $\begin{array}{l}\text { Optic } \\
\text { chiasm/nerves }\end{array}$ & 54 Gy & & $\begin{array}{l}\text { Can increase to 55-60Gy at clinician's } \\
\text { discretion/patient's consent }\end{array}$ \\
\hline Oral cavity & & & Aim for 45 Gy \\
\hline Cornea & & & Dmax 40 Gy \\
\hline Lacrimal gland & & & Dmax 30 Gy \\
\hline
\end{tabular}

VMAT plans using the Varian RapidArc technique (Varian Medical Systems, Palo Alto, CA) were planned using Eclipse Version 11.0.31 treatment planning software using the same CT-dataset and contoured volumes as the IMRT plans. A single arc technique was used with the gantry set to rotate through $360^{\circ}$ in a clockwise direction from a starting position of $181^{\circ}$ to a final position of $179^{\circ}$, and a double arc technique with the gantry set to rotate through $360^{\circ}$ in a clockwise direction from a starting position of $181^{\circ}$ to a final position of $179^{\circ}$, and through $360^{\circ}$ in a counterclockwise direction from a starting position of $179^{\circ}$ to a final position of $181^{\circ}$ according to the complexity of the phase being treated. The collimator rotation was individually optimized for each patient but generally set at $30^{\circ}$ and $330^{\circ}$ to reduce the effect of tongue-and-groove leakage (Figure 2).

The final dose calculation for all 3 of the plans, for each of the 10 patients, was performed using the anisotropic analytical algorithm (AAA) version 11.0 .31 with a $2.5 \mathrm{~mm}$ dose calculation grid space.

Treatment delivery for each of the 10 plans (3D-CRT, IMRT and VMAT) employed a Varian 21iX linear accelerator (Varian Medical Systems, Palo Alto, CA). All of the beams were delivered to a void bunker for IMRT and VMAT plans. 


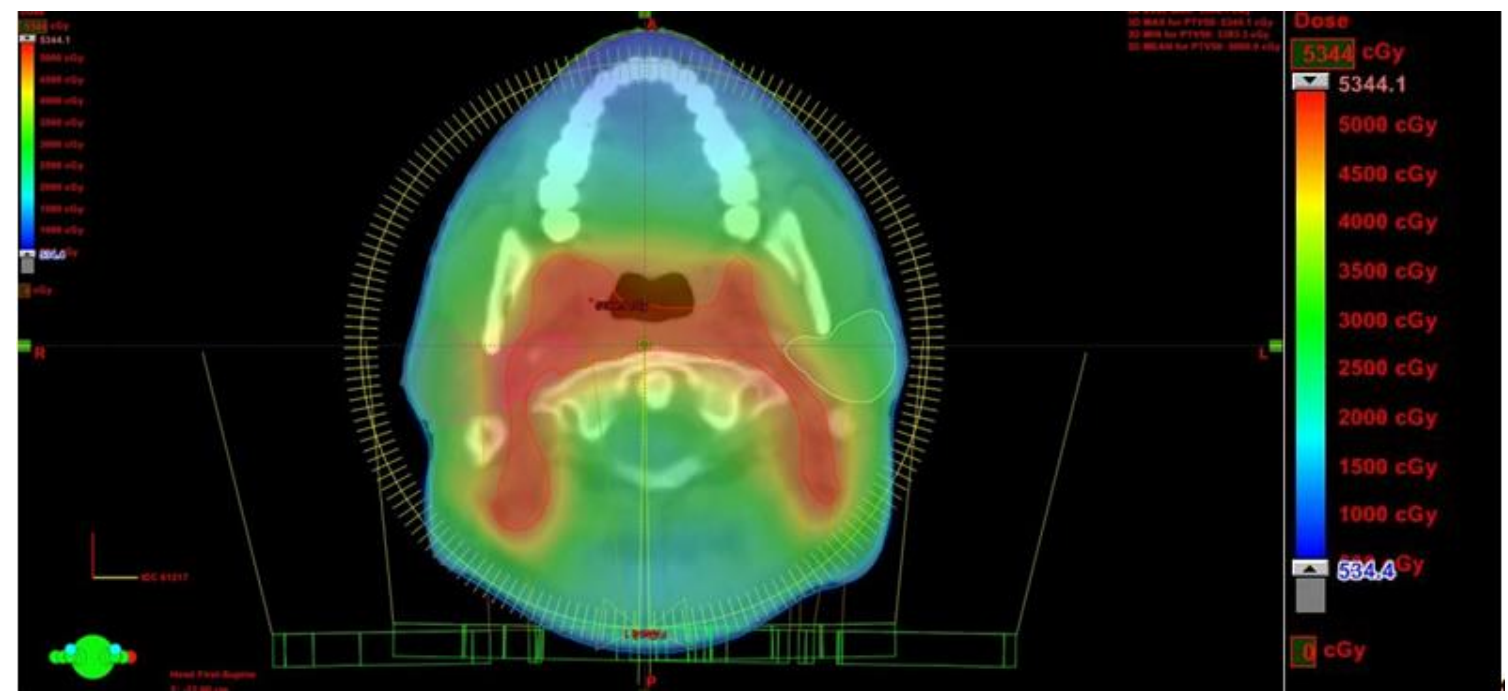

Fig. 2. Dose distribution (color-wash representation) for VMAT technique in a case of nasopharynx cancer

\section{Results}

Using 3D-CRT technique we obtained $\mathrm{D}_{\min }$ values between 1765.5-4074.3 cGy with an average of 3178.98 cGy, $D_{\max }$ between $5151.1-7314.8 \mathrm{cGy}$ with an average of 6907.64 cGy and $D_{\text {mean }}$ between 4094-5846.2 cGy with an average of 5596.57 cGy for the left parotid gland and $D_{\min }$ values between 1561.7- 4468.8 cGy with an average of 3279.16 cGy, $D_{\max }$ between 5169.5- 7340.1 cGy with an average of 6896.54 cGy and $D_{\text {mean }}$ between 3363.6-
6314.5 cGy with an average of 5579.3 cGy for the right parotid gland.

Using IMRT technique $D_{\min }$ decreases in average by $21.3 \%$ and $28.86 \%, D_{\max }$ decreases by $9.78 \%$ and $2 \%$ and $D_{\text {mean }}$ decreases by $15.89 \%$ and $13.88 \%$ for the left parotid and right parotid, respectively.

Using VMAT method $D_{\min }$ decreases in average by $27.63 \%$ and $24.80 \%, D_{\max }$ decreases by $1.41 \%$ and $0.97 \%$ and $D_{\text {mean }}$ decreases by $16.48 \%$ and $13.88 \%$ for left parotid left and right parotid, respectively (Table 2, Figure 3).

Table 2. Dosimetric values for the left and right parotids according to the irradiation techniques (STD is the standard deviation of the results, calculated by Eclipse treatment planning software)

\begin{tabular}{|c|c|c|c|c|c|c|c|c|c|c|c|c|c|c|c|c|c|c|}
\hline & \multicolumn{18}{|c|}{ Left parotid } \\
\hline & \multicolumn{4}{|c|}{ 3D-CRT } & \multicolumn{4}{|c|}{ IMRT } & \multicolumn{4}{|c|}{ VMAT } & \multicolumn{3}{|c|}{ IMRT(58) } & \multicolumn{3}{|c|}{ VMAT((8) } \\
\hline No & $\operatorname{Dmin}(G y)$ & Dmean(Gy) & $\operatorname{Dmax}(\mathrm{Gy})$ & STD(Gy) & $\operatorname{Dmin}(G y)$ & Dmean(Gy) & $\operatorname{Dmax}(G y) \mid \mathrm{s}$ & $\operatorname{STD}(G y)$ & $\operatorname{Dmin}(G y)$ & Dmean(Gy) & $|\mathrm{Dmax}(G y)| s$ & $\operatorname{STD}(G y)$ & Dmin & Dmean & $D \max$ & Dmin & Dmean & $D \max$ \\
\hline 1 & 17.66 & 40.94 & 51.51 & 8.37 & 15.04 & \begin{tabular}{ll|}
41.06 \\
\end{tabular} & \begin{tabular}{l|l}
64.47 \\
\end{tabular} & 8.52 & 16.83 & 41.63 & 64.30 & 8.18[ & -14.82 & 0.22[ & 25.16 & -4.62 & 1.70 & 24.83 \\
\hline 2 & 3425 & 60.88 & 70.88 & 8.03 & 30.80 & \begin{tabular}{l|l}
59.97 \\
\end{tabular} & \begin{tabular}{l|l}
72.55 \\
\end{tabular} & 9.41 & 27.55 & 55.98 & 72.39 & 11.79 & -10.05 & -1.50[ & 2.38 & -19.55 & -8.05 & 2.16 \\
\hline 3 & 33.79 & 78 & 70.72 & 6.86 & 22.22 & \begin{tabular}{l|l}
41.75 \\
\end{tabular} & 68.01 & 6.74 & 23.08 & 41.83 & 65.68 & 8.55[ & -34.24 & -17.78 & -6.67 & -31.69 & -17.62 & -7.13 \\
\hline 4 & 40.74 & 58.32 & 70.46 & 7.00 & 31.60 & \begin{tabular}{|l|l|l|}
51.68 & \\
\end{tabular} & \begin{tabular}{l|l|l|}
71.85 & \\
\end{tabular} & 9.77 & 26.96 & 51.53 & 71.39 & 10.86 & -22.45 & -11.38 & 1.97 & -33.83 & -11.64 & 1.32 \\
\hline 5 & 40.09 & 56.15 & 67.42 & 4.65 & 30.22 & 49.00 & 65.93 & 4.50 & 30.25 & 48.30 & \begin{tabular}{ll|l}
48.30 & \\
\end{tabular} & 6.36 . & -28.62 & -12.72 & -2.21 & -24.56 & $-13,97$ & -28.35 \\
\hline 6 & 20.33 & 58.12 & 72.46 & 9.05 & 12.06 & \begin{tabular}{|c|}
37.27 \\
\end{tabular} & \begin{tabular}{|c|}
72.76 \\
\end{tabular} & 12.39 & 11.04 & 40.00 & 71.19 & 12.69 & -40.69 & -35.8 & 0.41 & -45.69 & -31.18 & -1.75 \\
\hline 7 & 34.66 & 60.06 & 72.54 & 9.24 & 30.08 & 53.99 & $71.90 \mid$ & 10.04 & 28.17 & 51.94 & 73.14 & 10.85 & -13.23 & -10.11 & -0.88 & -18.74 & -13.52 & 0.83 \\
\hline 8 & 31.03 & .58 & 73.15 & 8.87 & 18.47 & 40.92 & 68.58 & 8.03 & 20.19 & 43.34 & 67.75 & 9.85 & -40.49 & -28.93 & -6.25 & -34.94 & -24.73 & $-7,38$ \\
\hline 9 & 31.90 & 46 & 69.23 & 6.62 & 22.46 & 49.08 & 19.13 & 11.18 & 19.13 & 44.31 & 71.73 & 9.52 & -29.60 & $-16,05$ & -72.36 & -40.02 & -24.21 & 3.62 \\
\hline 10 & 33.45 & 58.37 & 72.43 & 7.64 & 13.90 & \begin{tabular}{l|l}
43.87 & \\
\end{tabular} & \begin{tabular}{l|l}
43.87 & \\
\end{tabular} & 12.29 & 25.90 & \begin{tabular}{|l|l|}
45.78 \\
\end{tabular} & 70.77 & 11.96[ & -58.44 & -24.85 & -39.43 & -22.59 & -21.58 & -2.29 \\
\hline \multirow[t]{3}{*}{ Average } & 31.79 & 55.97 & 69.08 & & 22.68 & 46.86 & 61.70 & & 22.91 & 46.46 & 67.66 & & -28.86 & $\mid-15.89$ & -9.79 & -27.63 & -16.48 & -1.41 \\
\hline & \multicolumn{18}{|c|}{ Right parotid } \\
\hline & \multicolumn{4}{|c|}{ 3D-CRT } & \multicolumn{4}{|c|}{ IMRT } & \multicolumn{4}{|c|}{ VMAT } & \multicolumn{3}{|c|}{ IMRT( $(x)$} & \multicolumn{3}{|c|}{ VMATA(8) } \\
\hline No & $\operatorname{Dmin}\left(G_{y}\right)$ & Dmean(Gy) & $D \max (G y)$ & STD(Gy) & Dmin(Gy) & ean $(G y)$ & $\mid \mathrm{mmax}$ (Gy) & STD(Gy) & $\operatorname{Dmin}\left(G_{y}\right)$ & Dmean (Gy) & $|D \max (G y)|$ & STD(Gy) & Dmin & Dmean & $D_{\max }$ & Dmin & Dmean & Dmax \\
\hline 1 & 15.62 & 33.64 & 51.70 & 10.31 & 19.72 & 40.04 & 62.60 & 10.29 & 16.90 & 38.77 & 62.26 & 11.50 & 26.30 & 19.03 & 21.09 & 8.21 & 9.32 & 20.4 \\
\hline 2 & 33.83 & 59.82 & 70.19 & 8.71 & 29.61 & 55.60 & 72.31 & 12.03 & 23.01 & 54.88 & 71.73 & 12.45 & -12.47 & -7.05 & 3.02 & -31.97 & -8.27 & 2.18 \\
\hline 3 & 39.24 & 54.79 & 72.07 & 7.48 & 28.04 & 46.29 & 68.87 & 9.30 & 23.52 & 45.53 & 66.79 & $\begin{array}{ll}9.57 \\
\end{array}$ & -33.63 & -15.52 & -4.4 .4 & -40.05 & -16.89 & -7.32 \\
\hline 4 & 34.06 & 53.30 & 69.43 & 5.06 & 25.22 & 45.12 & 70.68 & 7.59 & 24.63 & 44.50 & 67.77 & 8.24 & -25.94 & -15.35 & 1.80 & -27.69 & -16.51 & -2.38 \\
\hline 5 & 37.81 & 57.83 & 67.95 & 5.86 & 34.44 & 51.47 & 67.55 & 6.57 & 36.15 & 53.78 & 68.64 & 6.98 & -8.91 & -11.00 & -0.58 & -4.90 & -7.01 & 1.02 \\
\hline 6 & 82 & .15 & 73.40 & 7.03 & 12.58 & 37.55 & 71.20 & 15.41 & 15.52 & 44.48 & 71.38 & 12.58 & $-56,33$ & -40.54 & -3.00 & -46.14 & -29.55 & -2.75 \\
\hline 7 & 31.93 & 55.94 & 69.13 & 8.62 & 27.51 & 53.47 & 70.37 & 10.65 & 18.46 & 47.25 & 69.09 & 11.08 & -13.85 & -4.41 & 1.79 & -42.18 & -15.54 & -0.06 \\
\hline 8 & 33.68 & 21 & 73.68 & 9.87 & 22.01 & 46.67 & 72.48 & 13.80 & 28.70 & 49.43 & 72.10 & 12.85 & $\begin{array}{ll}-34.65 \\
\end{array}$ & -21.18 & -1.6 & -20.74 & -16.5 & -2.12 \\
\hline 9 & .69 & 62.31 & 70.38 & 5.46 & 32.29 & 51.79 & 71.38 & 11,18 & 30.31 & 47.88 & 72.09 & 10.34 & -27.74 & -16.88 & 1.42 & -32.17 & -23.16 & 2.46 \\
\hline 10 & 28.25 & 57.95 & 71.77 & 11.34 & 20.95 & 45.73 & 72.18 & 13.54 & 25.17 & 49.43 & 70.48 & 12.68 & -25.8 & -21.08 & 0.57 & -10.89 & -14.70 & -1.81 \\
\hline erage & 32.79 & 55.79 & 68.97 & & 25.04 & 47.37 & 69.96 & & 24.04 & 47.39 & 70.48 & & -21.31 & -13.40 & 2.01 & -24.80 & -13.88 & 0.97 \\
\hline
\end{tabular}




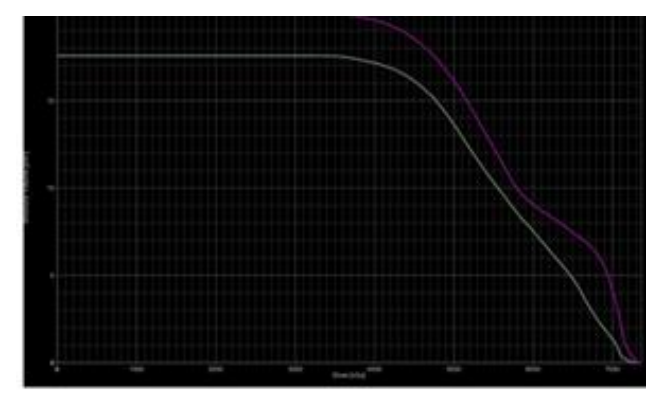

a)

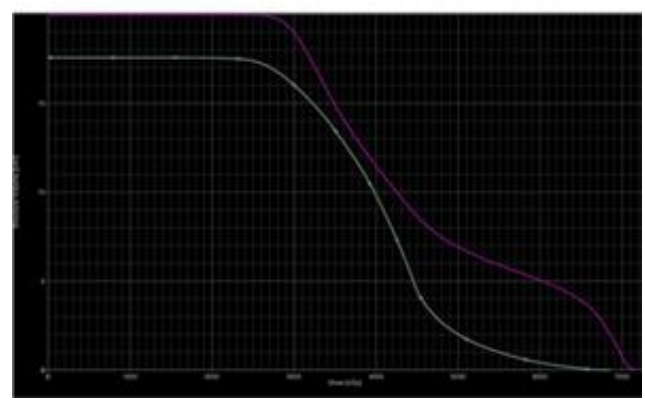

b)

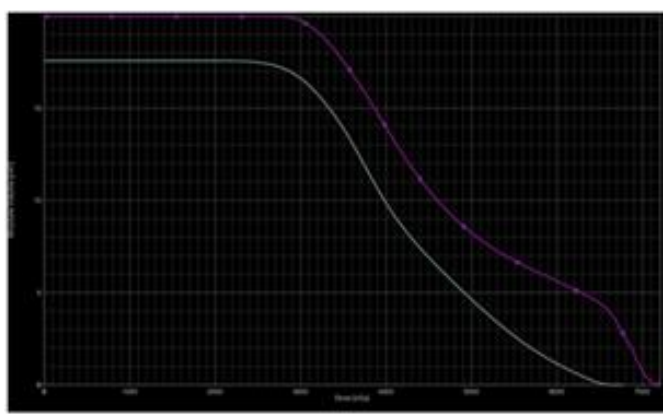

c)

Fig. 3. DVH curves for parotid (purple for right and green for left) glands for a) 3D; b) IMRT; and c) VMAT radiotherapy techniques, respectively

\section{Discussions}

More recent developed, VMAT technique derived from IMRT, using a dynamically multileaf collimator allows continuous, rotational irradiation with different dose rates, significantly decreasing time of irradiation and number of monitor units, increased patient comfort immobilized under the thermo-plastic mask and also brought a benefit in a better conformity of the irregularly shaped volumes. In terms of organs at risk VMAT technique has the advantages or the equivalency in report to the fixed beam IMRT technique, most authors reporting significant decreased doses to the eye and to the parotids $[4,6,7]$.
Morphological and functional changes of the parotid glands were observed after IMRT for nasopharyngeal cancer. Preservation of the contralateral parotid glands was only partly achieved. Late salivary dysfunction has been associated to the parotid mean dosage. Extreme xerostomia (characterized as longterm salivary function capacity of $<25 \%$ of standard) is typically kept away from if no less than one parotid organ is saved to a mean dose of less than roughly 20 Gy or if both organs are saved to a mean dose of less of around 25 Gy (mean dose). A lower parotid mean dose was connected with better functionality. Salving submandibular gland 
fundamentally diminishes the danger of radiation impelled xerostomia [8].

Shrinkage of the anatomical structures does not result in significant dosimetric difference in target volumes and OARs, except for the parotid gland (real mean dose increases by approximately $10 \%$ compared to the dose reported by the planning software). The association of re-planning after 4 weeks of treatment and reducing margins can provide up to a $30 \%$ decrease of mean dose in parotid. The parotid glands shrink more than the submandibular glands, increasing the risk of parotid insight into nodal target isodose $[9,10]$.

Wang and collaborators evaluate using 3 computer tomography (CT) sets before the treatment and at the $15^{\text {th }}$ and $25^{\text {th }}$ fraction during the treatment, distances between the lateral/medial aspects of parotid glands (PG) and midline at the level of odontoid process. GTV volume loss in this study led to the most significant change in position of the parotid glands during the first 3 weeks of treatment than between 3 and 5 weeks of treatment [11].

Yao and collaborators identify in nasopharyngeal cancer radiotherapy three OARs (parotid gland, cochlea and tympanic cavity) for which obtaining recommended dosimetric constraints is very difficult even using the IMRT technique [12].

For patients receiving radio-chemotherapy not only parotids but the mouth and submandibular glands and organs are involved in the development of xerostomia. Mean dose reductions $<40$ Gy for the oral cavity and $<50$ Gy for at least one of the submandibular

\section{References}

1. Chambers MS, Rosenthal DI, Weber RS, et al. Radiation-induced xerostomia. Head Neck 2007; 29(1):58-63.

2. Rubin $P$, Constine LS, Marks LB. ALERT Adverse Late Effects of Cancer Treatment: Volume 1: General Concepts and Specific Precepts, Volume 2: Normal Tissue Specific Sites and Systems, New York: Springer, 2014.

3. Rathod S, Gupta T, Ghosh-Laskar S, et al. Quality-of-life (QOL) outcomes in patients with head and neck squamous cell carcinoma (HNSCC) treated with intensity-modulated radiation therapy (IMRT) compared to three- glands should be considered as dosimetric constrictions with a prognostic value for developing the radio-induced xerostomia independent of lowering the doses received by the parotid glands in cancers of nasopharynx and oropharynx radiotherapy [13].

In a group of 52 patients one half of whom were treated by $2 \mathrm{D}$ and $3 \mathrm{D}$ techniques and the rest were treated by IMRT technique with simultaneous integrated boost, Moretto and collaborators obtained superior results in terms of late xerostomia for IMRT method even when using a moderate hypo-fractionated scheme (simultaneous integrated boost) [14].

The doses received by the parotid glands are the main predictor of late xerostomia but delimitation and sparing the oral cavity and submandibular glands defined as organs at risk can be an additional factor for preservation of salivary function. Re-planning after 3-5 weeks of treatment brings significant benefits in reducing the xerostomia and should be considered if possible.

\section{Conclusion}

Implementation of IMRT and VMAT modern techniques in radiotherapy of nasopharynx cancer has led to significant decrease of the doses received by the parotid glands even in the absence of applied dosimetry constrictions A new optimization could significantly reduce mean doses received by the parotid glands with a potential to reduce xerostomia. dimensional conformal radiotherapy (3D-CRT): evidence from a prospective randomized study. Oral Oncol 2013; 49(6):634-642.

4. Nishimura $Y$, Komaki R. Intensity-Modulated Radiation Therapy, Clinical Evidence and Techniques, Tokio: Springer, 2015.

5. Jensen SB, Pedersen AM, Vissink A, et al. A systematic review of salivary gland hypofunction and xerostomia induced by cancer therapies: prevalence, severity and impact on quality of life. Support Care Cancer 2010; 18(8):1039-1060. 
6. Ozdemir S, Coban Y, Akin M, et al. Dosimetric evaluation of nasopharyngeal carcinomas irradiated with different IMRT techniques. $J$ BUON 2014; 19(4):953-957.

7. Orlandi E, Giandini T, lannacone E, et al. Radiotherapy for unresectable sinonasal cancers: dosimetric comparison of intensity modulated radiation therapy with coplanar and non-coplanar volumetric modulated arc therapy. Radiother Oncol 2014; 113(2):260266.

8. Obinata $K$, Nakamura $M$, Carrozzo $M$, et al. Changes in parotid gland morphology and function in patients treated with intensitymodulated radiotherapy for nasopharyngeal and oropharyngeal tumors. Oral Radiol 2014; 30:135-141.

9. $\mathrm{Wu} Q$, Chi $\mathrm{Y}$, Chen PY, et al. Adaptive replanning strategies accounting for shrinkage in head and neck IMRT. Int J Radiat Oncol Biol Phys 2009; 75(3):924-932.

10. Wang $\mathrm{ZH}$, Yan $\mathrm{C}$, Zhang $\mathrm{ZY}$, et al. Radiationinduced volume changes in parotid and submandibular glands in patients with head and neck cancer receiving postoperative radiotherapy: a longitudinal study. Laryngoscope 2009; 119(10):1966-1974.

11. Wang $\mathrm{W}$, Yang $\mathrm{H}, \mathrm{Mi} Y$. Rules of parotid gland dose variations and shift during intensity modulated radiation therapy for nasopharyngeal carcinoma. Radiat Oncol 2015; 10:3.

12. Yao JJ, Chen FP, Zhou GQ, et al. A prospective study on radiation doses to organs at risk (OARs) during intensity-modulated radiotherapy for nasopharyngeal carcinoma patients. Oncotarget 2016; 7(16):21742-21752.

13. Little M, Schipper M, Feng FY, et al. Reducing xerostomia after chemo-IMRT for head-andneck cancer: beyond sparing the parotid glands. Int J Radiat Oncol Biol Phys 2012; 83(3):1007-1014.

14. Moretto $F$, Rampino $M$, Munoz $F$, et al. Conventional 2D (2D-RT) and 3D conformal radiotherapy (3D-CRT) versus intensitymodulated radiotherapy (IMRT) for nasopharyngeal cancer treatment. Radiol Med $2014 ; 119(8): 634-641$. 